\title{
Critical dynamics on a large human Open Connectome network
}

\author{
Géza Ódor* \\ Institute of Technical Physics and Materials Science, Centre for Energy Research of the Hungarian Academy of Sciences, \\ P.O. Box 49, H-1525 Budapest, Hungary
}

(Received 12 April 2016; revised manuscript received 17 July 2016; published 23 December 2016)

\begin{abstract}
Extended numerical simulations of threshold models have been performed on a human brain network with $N=836733$ connected nodes available from the Open Connectome Project. While in the case of simple threshold models a sharp discontinuous phase transition without any critical dynamics arises, variable threshold models exhibit extended power-law scaling regions. This is attributed to fact that Griffiths effects, stemming from the topological or interaction heterogeneity of the network, can become relevant if the input sensitivity of nodes is equalized. I have studied the effects of link directness, as well as the consequence of inhibitory connections. Nonuniversal power-law avalanche size and time distributions have been found with exponents agreeing with the values obtained in electrode experiments of the human brain. The dynamical critical region occurs in an extended control parameter space without the assumption of self-organized criticality.
\end{abstract}

DOI: 10.1103/PhysRevE.94.062411

\section{INTRODUCTION}

Theoretical and experimental research provides many signals for the brain to operate in a critical state between sustained activity and an inactive phase [1-5]. Critical systems exhibit optimal computational properties, suggesting why the nervous system would benefit from such mode [6]. For criticality, certain control parameters need to be tuned, leading to the obvious question: why and how this is achieved? This question is well known in statistical physics; the theory of self-organized criticality (SOC) of homogeneous systems has a long history since the pioneering work of [7]. In the case of competing fast and slow processes, SOC systems can self-tune themselves in the neighborhood of a phase transition point [8]. Many simple homogeneous models have been suggested to be described by power laws (PLs) and various other critical phenomena, very often without identifying SOC responsible processes. Alternatively, it has recently been proposed that living systems might also self-tune to criticality as a consequence of evolution and adaptation [9].

Real systems, however, are highly inhomogeneous and one must consider whether heterogeneity is weak enough to use homogeneous models. Heterogeneity is also called disorder in statistical physics and can lead to such rare-region (RR) effects that smear the phase transitions [10]. RRs can have various effects depending on their relevancy. They can change a discontinuous transition to a continuous one [11,12], or can generate so-called Griffiths phases (GPs) [13], or can completely smear a singular phase transition. In the case of GPs, critical-like power-law dynamics appears over an extended region around the critical point, causing slowly decaying autocorrelations and burstiness [14]. This behavior was proposed to be the reason for the working memory in the brain [15]. Furthermore, in GPs the susceptibility is infinite for an entire range of control parameters near the critical point, providing high sensitivity to stimuli, beneficial for information processing.

Therefore, studying the effects of heterogeneity is a very important issue in models of real systems, in particular in neuroscience. It has been conjectured that network heterogeneity

\footnotetext{
*odor@mfa.kfki.hu
}

can cause GPs if the topological (graph) dimension $D$, defined by $N_{r} \sim r^{D}$, where $N_{r}$ is the number of $(j)$ nodes within topological distance $r=d(i, j)$ from an arbitrary origin $(i)$, is finite [16]. This hypothesis was pronounced for the contact process (CP) [17], but subsequent studies found numerical evidence for its validity in the case of more general spreading models [18-20]. Recently, a GP has been reported in synthetic brain networks [21-23] with finite $D$. At first sight this seems to exclude relevant disorder effects in the so-called small-world network models. However, in finite systems PLs are observable in finite time windows for large random sample averages [24].

Very recently we have studied the topological behavior of large human connectome networks and found that contrary to the small-world network coefficients, they exhibit a topological dimension slightly above $D=3$ [25]. This suggests weak long-range connections, in addition to the $D=3$ dimensional embedding ones, and warrants seeing heterogeneity effects in dynamical models defined on them. These graphs contain link weight connection data, thus one can study the combined effect of topological and interaction disorder, assuming a quasistatic network.

This work provides a numerical analysis based on huge data sets of the Open Connectome Project (OCP) [26], obtained by diffusion tensor imaging (DTI) [27] to describe structural brain connectivity. Earlier studies of the structural networks were much smaller sized; for example, the one obtained by Sporns and collaborators, using diffusion imaging techniques $[28,29]$, consists of a highly coarse-grained mapping of anatomical connections in the human brain, comprising $N=998$ brain areas and the fiber tract densities between them. The graph used here comprises $N=848848$ nodes, allowing one to run extensive dynamical simulations on conventional or graphical processor clusters that can provide strong evidence for possible scaling behavior. It is essential to consider large connectomes, based on real experimental data, even if they are coarse grained and suffer from systematic errors and artifacts, because synthetic networks always rely on some subjective assumptions of the topologies. Smaller systems near a singularity point of a phase transition, where the correlations may diverge, suffer from finite size corrections, which can hide criticality or rare region effects. 


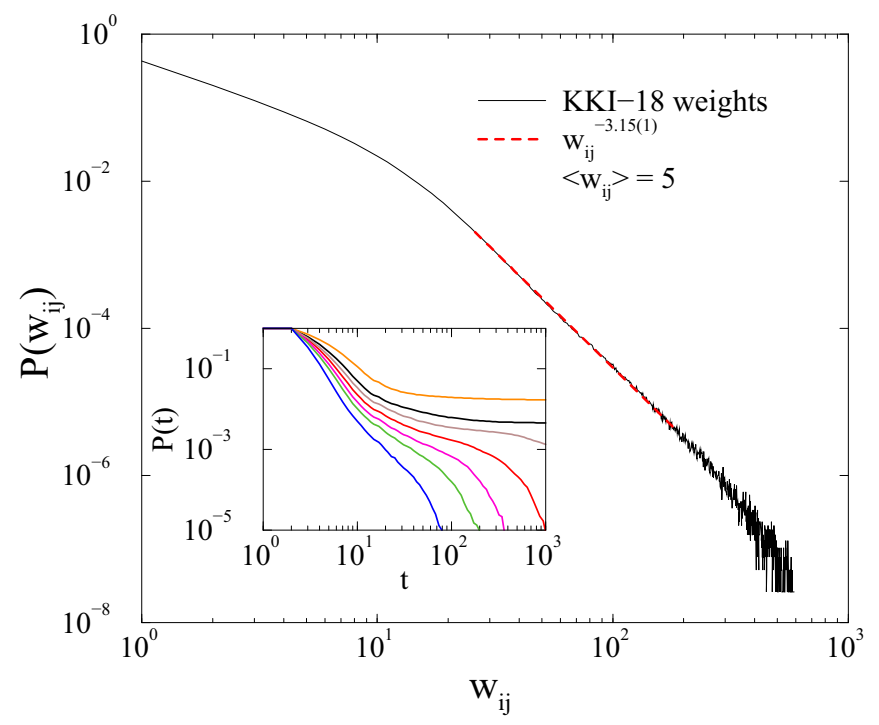

FIG. 1. Link weight probability density function of the KKI-18 OCP graph. Dashed line: a PL fit for intermediate $w_{i j}$ 's. Inset: survival probability in the $K=6$ threshold model near the transition point for $\lambda=0.003, v=0.3,0.4,0.45,0.5,0.55,0.6,0.7$ (top to bottom curves).

\section{MODELS AND METHODS}

Currently, connectomes can be estimated in humans at $1 \mathrm{~mm}^{3}$ scale, using a combination of diffusion weighted magnetic resonance imaging, functional magnetic resonance imaging, and structural magnetic resonance imaging scans. The large graph "KKI-18" used here is generated by the MIGRAINE method as described in [30]. Note that OCP graphs are symmetric, weighted networks obtained by image processing techniques, where the weights measure the number of fiber tracts between nodes. The investigated graph exhibits a single giant component of size $N=836733$ nodes (out of $N=848848$ ) and several small subcomponents, ignored here, to disregard an unrealistic brain network scenario. This graph has 41523931 undirected edges, but to make it more realistic, first I studied a diluted one, in which $20 \%$ of the edges were changed to directed by a random connection removal process. This directionality value is in between 5/126 of [31] and 33\% reported in [32]. I shall discuss the relevancy of this edge asymmetry assumption.

Weights between nodes $i$ and $j$ of this graph vary between 1 and 854 and the probability density function is shown in Fig. 1. Following a sharp drop one can observe a PL region for $20<$ $w_{i j}<200$ with cutoff at large weights. The average weight of the links is $\simeq 5$. Note that the average degree of the KKI-18 graph is $\langle k\rangle=156$ [25], while the average of the sum of the incoming weights of nodes is $\left\langle W_{i}\right\rangle=1 / N \sum_{i} \sum_{j} w_{i j}=448$.

A two-state $\left(x_{i}=0\right.$ or 1$)$ dynamical spreading model was used to describe the propagation, branching, and annihilation of activity on the network. This threshold model is similar to those of Refs. [5,33]. The dynamical process is started by activating a randomly selected node. At each network update every $(i)$ node is visited and tested if the sum of incoming weights $\left(w_{i, j}\right)$ of active neighbors reaches a given threshold value

$$
\sum_{j} x_{j} w_{i, j}>K
$$

If this condition is satisfied a node activation is attempted with probability $\lambda$. Alternatively, an active node is deactivated with probability $v$. New states of the nodes are overwritten only after a full network update, i.e., a synchronous update is performed at discrete time steps. The updating process continues as long as there are active sites or up to a maximum time limit $t=10^{5}$ Monte Carlo sweeps (MCS). In the case in which the system falls into inactivity the actual time step is stored in order to calculate the survival probability $P(t)$ of runs. The average activity: $\rho(t)=1 / N \sum_{i=1}^{N} x_{i}$ and the number of activated nodes during the avalanche $s=\sum_{i=1}^{N} \sum_{t=1}^{T} x_{i}$ of duration $T$ is calculated at the end of the simulations. This stochastic cellular automaton type of updating is not expected to affect the dynamical scaling behavior [23] and provides a possibility for network-wise parallel algorithms. Measurements on $10^{5}-10^{7}$ independent runs, started from randomly selected, active initial sites were averaged over at each control parameter value.

By varying the control parameters $K, \lambda$, and $v$, I attempted to find a critical point between an active and an absorbing steady state. At a critical transition point the survival probability is expected to scale asymptotically as

$$
P(t) \propto t^{-\delta},
$$

where $\delta$ is the survival probability exponent [34]. This can be related to the avalanche (total number of active sites during the spreading experiment) duration scaling: $p(t) \propto t^{-\tau_{t}}$, via the relation $\tau_{t}=1+\delta$ [35]. In seed experiments the number of active sites initially grows as

$$
N(t) \propto t^{\eta}
$$

with the exponent $\eta$, related to the avalanche size distribution $p(s) \propto s^{-\tau}$, via the scaling law

$$
\tau=(1+\eta+2 \delta) /(1+\eta+\delta)
$$

[35]. To see corrections to the scaling I also determined the local slopes of the dynamical exponents $\delta$ and $\eta$ as the discretized, logarithmic derivative of (2) and (3). The effective exponent of $\delta$ is measured as

$$
\delta_{\mathrm{eff}}(t)=-\frac{\ln P(t)-\ln P\left(t^{\prime}\right)}{\ln (t)-\ln \left(t^{\prime}\right)},
$$

using $t-t^{\prime}=8$, and similarly can one define $\eta_{\mathrm{eff}}(t)$. This difference selection has been found to be optimal in noise reduction versus effective exponent range [36].

As the OCP graph is very inhomogeneous, it appears that for a given set of control parameters only the hub nodes can be activated and the weakly coupled ones do not play any role. This is rather unrealistic and is against the local sustained activity requirement for the brain [33]. Indeed, there is some evidence that neurons have a certain adaptation to their input excitation levels [37] and can be modeled by variable 
thresholds [38]. This adaptation leading to some homeostasis can be assumed to be valid on the coarse-grained node level too. To model nodes with variable thresholds of equalized sensitivity, I modified the incoming weights by normalizing them as $w_{i, j}^{\prime}=w_{i, j} / \sum_{j \in \text { neighb. of } i} w_{i, j}$. Although the update rules are unchanged, I shall call such simulations "variable threshold model" studies.

\section{DYNAMICAL SIMULATION RESULTS}

First I summarize the results for the related homogeneous system. It is well known that in branching and annihilating models with multiparticle $(A)$ reactions $m A \rightarrow(m+$ $k) A, n A \rightarrow(n-l) A$ for $m>n$ the phase transition in the high dimensional, mean-field limit is first order type [39]. Considering the sum of occupied neighbors as the incoming activation potential, there is a sudden change in the balance of activation and deactivation possibilities as we approach the absorbing phase, since annihilation can occur unconditionally. Therefore, it can be expected that for threshold models with $K>1$, near and above the upper critical dimension, which is expected to be $d_{c} \leqslant 4$, we observe discontinuous transitions. First I run the threshold model on an unweighted three-dimensional lattice with $N=10^{6}$ nodes and periodic boundary conditions. I tested the low $K=2,3,6$ threshold cases, being the most possible candidates for the occurrence of PL dynamics. However, for high branching probability $(\lambda=1)$, where an efficient neural network should work, an exponentially fast evolution to the inactive state occurred for any $v>0.001$. On the other hand, for $v \rightarrow 0$ the survival probability remains finite, but the transition is very sharp-in agreement with the results of [39] - and we cannot find PL dynamics.

In the case of various quenched heterogeneities, a similar model, the $\mathrm{CP}$, has recently been studied on three-dimensional lattices with diluted disorder in [40]. Extensive computer simulations gave numerical evidence for nonuniversal power laws typical of a Griffiths phase as well as activated scaling at criticality. The disorder, generated by the addition of long-range connections, has also been found to be relevant in the case of the CP and threshold models in one [16] and two dimensions [23], provided the probability of connections fell quickly enough, i.e., $p \propto r^{-s}$, with $s \geqslant 2 D$. This means that in the case of a $D=3$ system, the graph dimension remains finite and a GP may be expected if $s \geqslant 6$. However, in a real connectome we cannot investigate these heterogeneities separately.

\section{A. Threshold model}

Next, I performed simulations of the threshold model on the KKI-18 graph with $K=1,2,6$, since for larger $K$ 's we do not expect criticality. Again, temporal functions did not show PL behavior. Instead, one can observe an exponentially fast drop of $P(t)$ to zero or to some finite value, depending on the control parameters. Discontinuous transition occurs at very low $\lambda$ 's, as shown in the inset of Fig. 1. Therefore, the heterogeneity of the OCP graph is not strong enough to round the discontinuous transition, observed on the homogeneous lattice unlike in the case of the models in $[11,12]$. It appears that hubs, with large

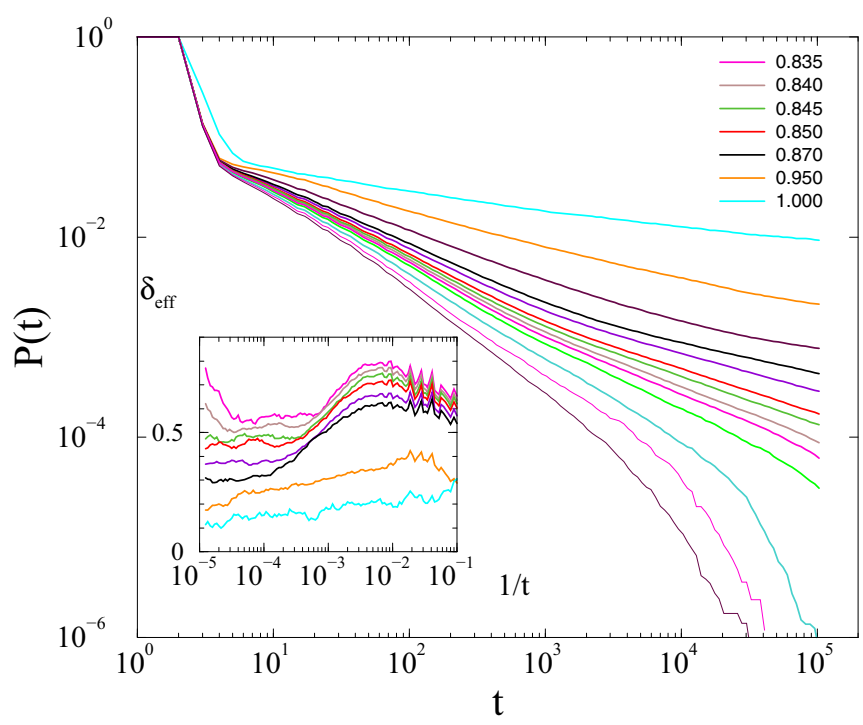

FIG. 2. Avalanche survival distribution of the relative threshold model with $K=0.25$, for $v=0.95$ and $\lambda=0.8,0.81$, $0.82,0.83,0.835,0.84,0.845,0.85,0.86,0.87,0.9,0.95,1$ (bottom to top curves). Inset: Local slopes of the same from $\lambda=0.835$ to $\lambda=1$ (top to bottom curves). Griffiths effect manifests by slopes reaching a constant value as $1 / t \rightarrow 0$.

$W_{i}=\sum_{j} w_{i j}$, determine the behavior of the whole system, while other nodes do not play a role. These hubs keep the system active or inactive, ruling out the occurrence of local RR effects as in the case of infinite dimensional networks [24].

\section{B. Variable threshold model}

To test this, I turned towards the simulations of variable threshold models. The control parameter space was restricted by fixing $\lambda \simeq 1$, which mimics an efficient brain model. Transitions could be found for $K<0.5$; for higher thresholds the models evolve to an inactive phase for any $v$ 's. For the time being I set $K=0.25$. Figure 2 suggests a phase transition at $v=0.95$ and $\lambda=0.88(2)$, above which $P(t)$ curves evolve to finite constant values. It is very hard to locate the transition clearly, since the evolution slows down and $\log$-periodic oscillations are also added (see inset of Fig. 2). The straight lines on the logarithmic plot of $\delta_{\text {eff }}$ at $\lambda \simeq 1$ suggest ultraslow dynamics as in the case of a strong disorder fixed point [10]. Indeed, a logarithmic fitting at $\lambda=0.88$ results in $P(t) \simeq \ln (t)^{-3.5(3)}$, which is rather close to the the three-dimensional strong disorder universal behavior [40,41]. Simulations started from fully active sites show analogous decay curves for the density of active sites $\rho(t)$, expressing a rapidity reversal symmetry, characteristic of the directed percolation (DP) universality class [36], governing the critical behavior of such models [42]. However, for the graph dimension of this network, $D \simeq 3.2$, one should see $\delta>0.73$ in the case of DP universality [36], which can be excluded by the present simulations.

Below the transition point for fixed $v=0.95$ we can find $P(t)$ decay curves with PL tails, characterized by the exponents $0<\delta<0.5$, as we vary $\lambda$ between 0.845 and 0.88 . In this region the avalanche size distributions also show PL 


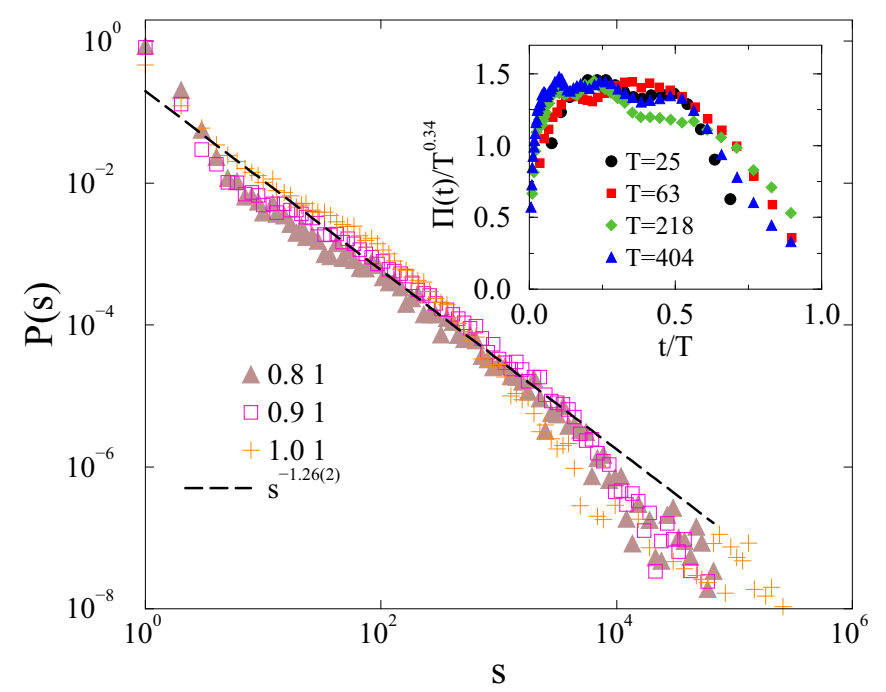

FIG. 3. Avalanche size distribution of the relative threshold model with $K=0.25$, for $v=1$ and $\lambda=1,0.9,0.8$. Dashed line: PL fit to the $\lambda=0.8$ case. Inset: Avalanche shape collapse for $T=25,63,218,404$ at $\lambda=0.86$ and $v=0.95$.

decay (Fig. 3), modulated by some oscillations due to the modular network structure, but the exponents of the curves are around $\tau=1.26(2)$, a smaller value than obtained by the brain experiments, $\tau \simeq 1.5[1]$.

\section{Avalanche average shape}

I have also tested the collapse of averaged avalanche distributions $\Pi(t)$ of fixed temporal sizes $T$ as in [43]. The inset of Fig. 3 shows good a collapse, obtained for avalanches of temporal sizes $T=25,63,218,404$ and using a vertical scaling $\Pi(t) / T^{0.34}$, which is near to the experimental findings reported in [43]. Note the asymmetric shapes, which are also in agreement with the experiments and could not be reproduced by the model of Ref. [43].

\section{Undirected links}

To test the robustness of the results, simulations were also run on the KKI-18 network with unidirectional edges at $K=0.25$. Similar PL tails have been obtained as before, but for the same control parameters the slopes of $\ln [P(\ln t)]$ curves were bigger, meaning that in the symmetric networks $\tau_{t}=1.4-1.7$ (see Fig. 4) and the avalanche size distributions also fell faster, characterized by $1.5<\tau<2$.

\section{E. Inhibitory connections}

In real brain networks inhibitory connections also happen. To model this I changed the sign of a certain portion of the weights randomly at the beginning of the simulations, i.e., $w_{i, j}^{\prime}=-w_{i, j}^{\prime}$. This produces further heterogeneity, thus stronger RR effects. Figure 5 shows the survival probabilities, when $30 \%$ of the links became inhibitory for $K=0.1$ and $\lambda=0.95$. The critical point, above which $P(t)$ signals persistent activity, is around $v=0.57$, very hard to locate clearly, since the evolution slows down and exhibits strong (oscillating)

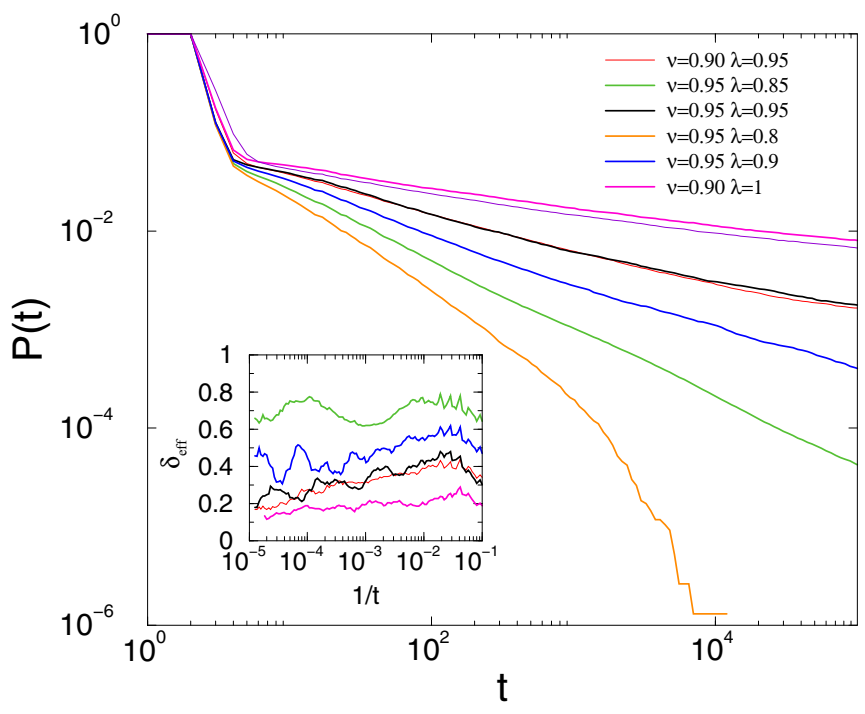

FIG. 4. The same as Fig. 2 in the case of the undirected graph. Inset: Local slopes of the curves.

corrections. Below the transition point the survival exponent changes continuously in the range $0<\delta<0.5$ as a response to the variation of $v$ between 0.5 and 0.57 (inset of Fig. 5). The corresponding avalanche size distributions (Fig. 6) exhibit PL tails with the exponent $\tau \simeq 1.5$, close to the experimental value for the brain [1]. A slight change of $\tau$ can also be observed by varying the control parameter below the critical point. This variation can be seen even better with the exponent $\eta$, related to $\tau$ via Eq. (4) (inset of Fig. 6), suggesting Griffiths effects.

For $20 \%$ of the inhibitory links the same $\tau$ 's were obtained, while $10 \%$ of inhibition resulted in $\tau \simeq 1.3$ near the critical point. For higher threshold values $(K=0.2,0.25)$ the critical

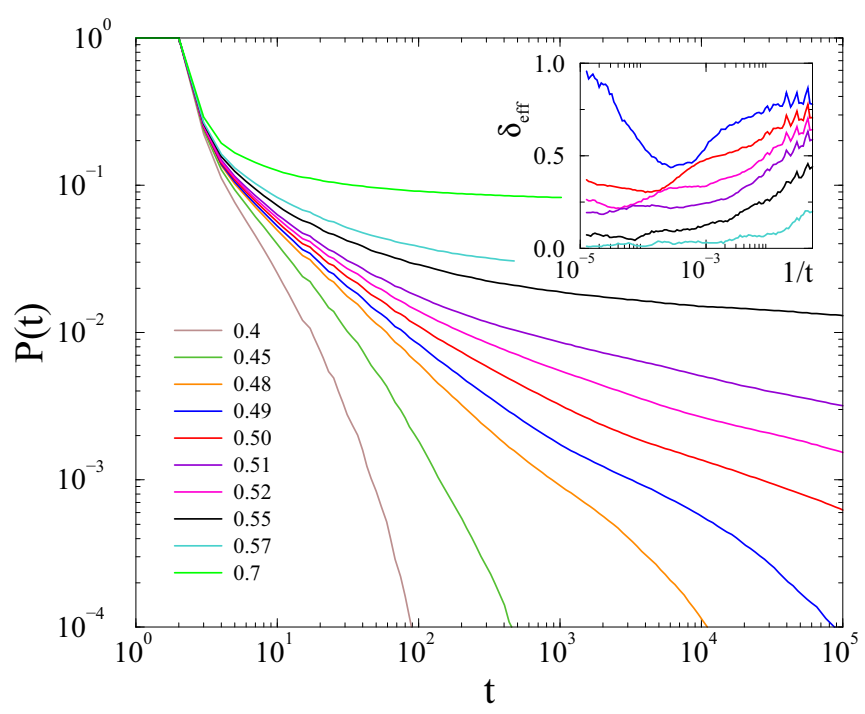

FIG. 5. Avalanche survival distribution of the relative threshold model with $30 \%$ inhibitory links at $K=0.1$, for $\lambda=0.95$ and $v=$ $0.4,0.45,0.49,0.5,0.51,0.52,0.550 .57,0.7$ (bottom to top curves). Inset: Local slopes of the same curves in opposite order. 


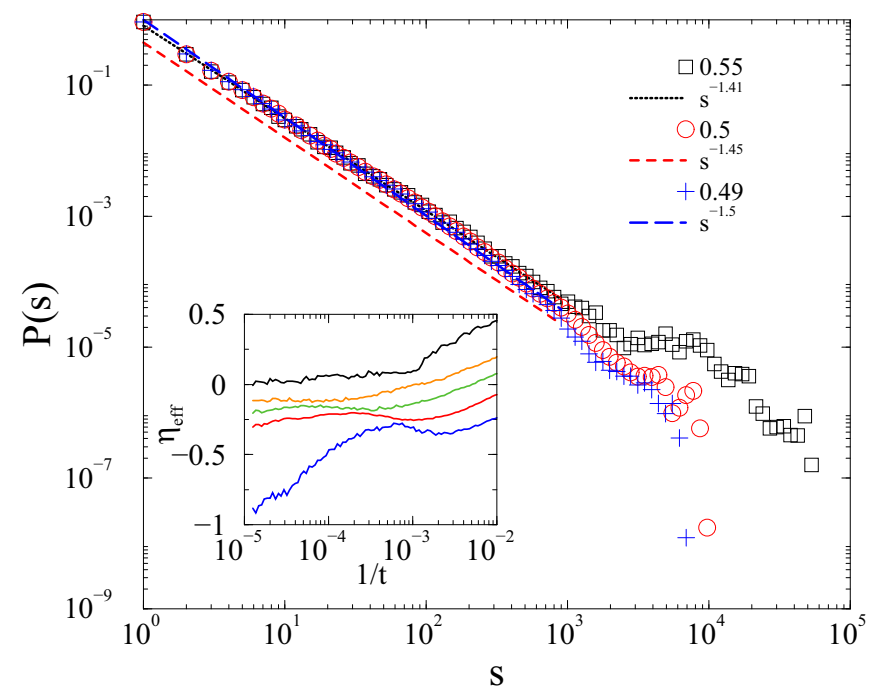

FIG. 6. Avalanche size distribution of the relative threshold model with $30 \%$ inhibitory links at $K=0.1, v=0.95$, and $\lambda=$ $0.49,0.5,0.55$. Dashed lines: PL fits. Inset: Effective $\eta$ exponent for $v=0.95$ and $\lambda=0.49,0.5,0.51,0.51,0.55$ (bottom to top curves).

point shifts to smaller $v$ parameters but Griffiths effects are still visible. However, avalanche size distributions exhibit faster decay, characterized by $\tau \simeq 1.7-2$.

\section{DISCUSSION AND CONCLUSIONS}

Neural variability makes the brain more efficient [44], therefore one must consider its effect in modeling. To study this, large-scale dynamical simulations have been performed on a human connectome model. The heterogeneities of an OCP graph are too weak to change the dynamical behavior of the threshold model of a homogeneous three-dimensional lattice. This seems to be true for other spreading models, such as the contact process [45]. In relative threshold models, defined by normalizing the incoming weights, Griffiths effects have been found for extended control parameter spaces. The inclusion of a $20 \%$ edge directness does not affect the results qualitatively, reflecting insensitivity to some potential artifacts of DTI, such as polarity detection. Random removal of connections emulates the effect of (unknown) noise in the data generation and since the majority of the edges are short, this procedure results in a relative enhancement of long connections, which is known to be underestimated by the DTI [46]. Scaling exponents on undirected OCPs vary in the range $1.4<\tau_{t}<1.7$ and $1.5<\tau<2$, close to neural experimental values [1].

The effects of other less relevant systematic errors and artifacts have not been investigated here. Radial accuracy affects, for example, the end point of the tracts, thus it influences the hierarchical levels of cortical organization [47]. The present OCP exhibits hierarchical levels by construction from the Desikan regions with (at least) two quite different scales. Preliminary studies [45] suggest that RR effects are enhanced by modularity, but not too much by the hierarchy. Transverse accuracy determines which cortical area is connected to which other. However, achieving fine-grained transverse accuracy is difficult for DTI, not only because of limited spatial resolution, but also because present measures are noisy and indirect. We may expect that the lack of precise connection pathways is not relevant for Griffiths effects as long as they do not affect the graph dimension, for example.

The introduction of $20 \%-30 \%$ of inhibitory links, selected randomly, results in Griffiths effects with avalanche size and time exponents, which scatter around the experimental figures. The exponents depend slightly on the control parameters as the consequence of RR effects. Strong and oscillating corrections to scaling are also observable as the result of the modular structure of the connectome.

As an earlier study [25] showed a certain level of universality in topological features (degree distributions, graph dimensions, clustering, and small-world coefficients of the OCP graphs), one can expect the same dynamical behavior and Griffiths effects of these models on OCP graphs in general. This expectation is supported further by the robustness of the results for random changing of the network details: inhibitory links, directedness or loss of connections up to $20 \%$. Therefore, one can safely take it for granted that the investigated connectome well describes similar ones available currently and can be considered as a useful prototype for numerical analysis.

It is important to note that while some rough tuning of the control parameters might be necessary to get closer to the critical point, one can see dynamical criticality even below a phase transition point without external activation, which is a safe expectation for brain systems [48]. Recent experiments suggest slightly subcritical brain states in vivo, devoid of dangerous overactivity linked to epilepsy.

One may debate the assumption of relative thresholds, which was found to be necessary to see slow dynamics. This introduces disassortativity, enhancing RR effects [19], besides the modularity [23]. However, inhibitory links increase the heterogeneity so drastically, that a full equalization of the internal sensitivity may not be an obligatory condition for finding Griffiths effects. This will be the target of further studies.

Probably the most important result of this study is that negative weights enable local sustained activity and promote strong RR effects without network fragmentation. Thus, connectomes with high graph dimensions can be subject to RR effects and can show measurable Griffiths effects. Another important observation is that PLs may occur in a single network, without sample averaging, due to the modular topological structure.

The codes and the graph used here are available by request from the author.

\section{ACKNOWLEDGMENTS}

I thank C. C. Hilgetag and R. Juhász for their useful discussions and M. A. Muñoz and M. T. Gastner for their comments. Support from the Hungarian research fund OTKA (Grant No. K109577) is acknowledged. 
[1] J. M. Beggs and D. Plenz, Neuronal avalanches in neocortical circuits, J. Neurosci. 23, 11167 (2003).

[2] C. Tetzlaff, S. Okujeni, U. Egert, F. Wörgötter, and M. Butz, Selforganized criticality in developing neuronal networks, PLoS Comput. Biol. 6, e1001013 (2010).

[3] G. Hahn et al., Neuronal avalanches in spontaneous activity in vivo, J. Neurophysiol. 104, 3312 (2010).

[4] T. L. Riberio et al., Spike avalanches exhibit universal dynamics across the sleep-wake cycle, PLoS One 5, e14129 (2010).

[5] A. Haimovici, E. Tagliazucchi, P. Balenzuela, and D. R. Chialvo, Brain Organization into Resting State Networks Emerges at Criticality on a Model of the Human Connectome, Phys. Rev. Lett. 110, 178101 (2013).

[6] For a review, see R. Legenstein and W. Maass, New Directions in Statistical Signal Processing: From Systems to Brain, edited by S. Haykin, J. C. Principe, T. Sejnowski, and J. McWhirter (MIT Press, Cambridge, 2007), pp. 127-154.

[7] P. Bak, C. Tang, and K. Wiesenfeld, Phys. Rev. A 38, 364 (1988).

[8] G. Pruessner, Self organized criticality (Cambridge University Press, Cambridge, 2012).

[9] J. Hidalgo et al., Information-based fitness and the emergence of criticality in living systems, Proc. Natl. Acad. Sci. USA 111, 10095 (2014).

[10] T. Vojta, Rare region effects at classical, quantum and nonequilibrium phase transitions, J. Phys. A 39, R143 (2006).

[11] P. M. Villa Martin, J. A. Bonachela, and M. A. Muñoz, Quenched disorder forbids discontinuous transitions in nonequilibrium low-dimensional systems, Phys. Rev. E 89, 012145 (2014).

[12] P. M. Villa Martin, M. Moretti, and M. A. Muñoz, Rounding of abrupt phase transitions in brain networks, J. Stat. Mech. (2015) P01003.

[13] R. B. Griffiths, Nonanalytic Behavior Above the Critical Point in a Random Ising Ferromagnet, Phys. Rev. Lett. 23, 17 (1969).

[14] G. Ódor, Slow, bursty dynamics as a consequence of quenched network topologies, Phys. Rev. E 89, 042102 (2014).

[15] S. Johnson, J. J. Torres, and J. Marro, Robust short-term memory without synaptic learning, PLoS One 8, e50276 (2013).

[16] M. A. Muñoz, R. Juhász, C. Castellano, and G. Ódor, Griffiths Phases on Complex Networks, Phys. Rev. Lett. 105, 128701 (2010).

[17] T. E. Harris, Contact interactions on a lattice, Ann. Probab. 2, 969 (1974).

[18] G. Ódor and R. Pastor-Satorras, Slow dynamics and rare-region effects in the contact process on weighted tree networks, Phys. Rev. E 86, 026117 (2012).

[19] G. Ódor, Rare regions of the susceptible-infected-susceptible model on Barabasi-Albert networks, Phys. Rev. E 87, 042132 (2013).

[20] G. Ódor, Spectral analysis and slow spreading dynamics on complex networks, Phys. Rev. E 88, 032109 (2013).

[21] P. Moretti and M. A. Muñoz, Griffiths phases and the stretching of criticality in brain networks, Nat. Commun. 4, 2521 (2013).

[22] P. Villegas, P. Moretti, and Miguel A. Muñoz, Frustrated hierarchical synchronization and emergent complexity in the human connectome network, Sci. Rep. 4, 5990 (2014)

[23] G. Ódor, R. Dickman, and G. Ódor, Griffiths phases and localization in hierarchical modular networks, Sci. Rep. 5, 14451 (2015).
[24] W. Cota, S. C. Ferreira, and G. Ódor, Griffiths effects of the susceptible-infected-susceptible epidemic model on random power-law networks, Phys. Rev. E 93, 032322 (2016).

[25] M. T. Gastner and G. Ódor, The topology of large Open Connectome networks for the human brain, Sci. Rep. 6, 27249 (2016).

[26] See http://www.openconnectomeproject.org

[27] Bennett A. Landman et al., Multi-parametric neuroimaging reproducibility: A 3-T resource study, NeuroImage 54, 2854 (2011).

[28] P. Hagmann et al., Mapping the structural core of human cerebral cortex, PLoS Biol. 6, e159 (2008).

[29] C. J. Honey et al., Predicting human resting-state functional connectivity from structural connectivity, Proc. Natl. Acad. Sci. USA 106, 2035 (2009).

[30] W. G. Roncal et al., MIGRAINE: MRI graph reliability analysis and inference for connectomics, Global Conference on Signal and Information Processing (GlobalSIP), 2013 IEEE (IEEE, New York, 2013).

[31] D. J. Felleman and D. C. Van Essen, Distributed hierarchical processing in the primate cerebral cortex, Cerebr. Cortex 1, 1 (1991).

[32] N. T. Markov et al., A weighted and directed interareal connectivity matrix for macaque cerebral cortex, Cerebr. Cortex 24, 17 (2012).

[33] M. Kaiser and C. C. Hilgetag, Optimal hierarchical modular topologies for producing limited sustained activation of neural networks, Front. Neuroinf. 4, 8 (2010).

[34] P. Grassberger and A. de la Torre, Reggeon field theory (Schlögl's first model) on a lattice: Monte Carlo calculations of critical behaviour, Ann. Phys. (NY) 122, 373 (1979).

[35] M. A. Muñoz, R. Dickman, A. Vespignani, and S. Zapperi, Avalanche and spreading exponents in systems with absorbing states, Phys. Rev. E 59, 6175 (1999).

[36] G. Ódor, Universality classes in nonequilibrium lattice systems, Rev. Mod. Phys. 76, 663 (2004).

[37] R. Azouz and C. M. Gray, Dynamic spike threshold reveals a mechanism for synaptic coincidence detection in cortical neurons in vivo, Proc. Natl. Acad. Sci. USA 97, 8110 (2000).

[38] M.-T. Hütt, M. K. Jain, C. C. Hilgetag, and A. Lesne, Stochastic resonance in discrete excitable dynamics on graphs, Chaos, Solitions Fractals 45, 611 (2012).

[39] G. Ódor, Phase transition classes in triplet and quadruplet reaction-diffusion models, Phys. Rev. E 67, 056114 (2003).

[40] T. Vojta, Monte Carlo simulations of the clean and disordered contact process in three dimensions, Phys. Rev. E 86, 051137 (2012).

[41] I. A. Kovács and F. Iglói, Infinite-disorder scaling of random quantum magnets in three and higher dimensions, Phys. Rev. B 83, 174207 (2011).

[42] J. Marro and R. Dickman, Nonequilibrium Phase Transitions in Lattice Models (Cambridge University Press, Cambridge, 1999).

[43] N. Friedman, S. Ito, B. A. W. Brinkman, M. Shimono, R. E. Lee DeVille, K. A. Dahmen, J. M. Beggs, and T. C. Butler, Universal Critical Dynamics in High Resolution Neuronal Avalanche Data, Phys. Rev. Lett. 108, 208102 (2012). 
[44] G. Orbán, P. Berkes, J. Fiser, and M. Lengyel, Neural variability and sampling-based probabilistic representations in the visual cortex, Neuron 92, 530 (2016).

[45] G. Ódor (unpublished).

[46] Saad Jbabdi and Heidi Johansen-Berg, Tractography: Where do we go from here?, Brain Connect. 1, 169 (2011).
[47] C. C. Hilgetag, M. A. ONeill, and M. P. Young, Hierarchical organization of macaque and cat cortical sensory systems explored with a novel network processor, Philos. Trans. R. Soc. London, Ser. B 355, 71 (2000).

[48] V. Priesemann et al., Spike avalanches in vivo suggest a driven, slightly subcritical brain state, Front. Syst. NeuroSci. 8, 108 (2014). 